\title{
The performance of hybrid ARIMA-GARCH modeling in forecasting gold price
}

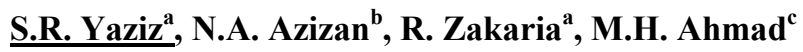 \\ ${ }^{a}$ Faculty of Industrial Sciences \& Technology, Universiti Malaysia Pahang. \\ ${ }^{b}$ Faculty of Technology, Universiti Malaysia Pahang. \\ ${ }^{c}$ Department of Mathematics, Faculty of Science, Universiti Teknologi Malaysia \\ Email: roslindar@ump.edu.my
}

\begin{abstract}
Gold has been considered a safe return investment because of its characteristic to hedge against inflation. As a result, the models to forecast gold must reflect its structure and pattern. Gold prices follow a natural univariate time series data and one of the methods to forecast gold prices is Box-Jenkins, specifically the autoregressive integrated moving average (ARIMA) models. This is due to its statistical properties, accurate forecasting over a short period of time, ease of implementation and able to handle nonstationary data. Despite the fact that ARIMA is powerful and flexible in forecasting, however it is not able to handle the volatility and nonlinearity that are present in the data series. Previous studies showed that generalized autoregressive conditional heteroskedatic $(\mathrm{GARCH})$ models are used in time series forecasting to handle volatility in the commodity data series including gold prices. Hence, this study investigate the performance of hybridization of potential univariate time series specifically ARIMA models with the superior volatility model, GARCH incorporates with the formula of Box-Cox transformation in analyzing and forecasting gold price. The Box-Cox transformation is used as the data transformation due to its power in normalizing data, stabilizing variance and reducing heteroskedasticity.
\end{abstract}

There is two-phase procedure in the proposed hybrid model of ARIMA and GARCH. In the first phase, the best of the ARIMA models is used to model the linear data of time series and the residual of this linear model will contain only the nonlinear data. In the second phase, the GARCH is used to model the nonlinear patterns of the residuals. This hybrid model which combines an ARIMA model with GARCH error components is applied to analyze the univariate series and to predict the values of approximation. In this procedure, the error term $\varepsilon_{t}$ of the ARIMA model is said to follow a GARCH process of orders $r$ and $s$.

The performance of the proposed hybrid model is analyzed by employing similar 40 daily gold price data series used by Asadi et al. (2012), Hadavandi et al. (2010), Khashei et al. (2009) and Khashei et al. (2008). From the plotting in-sample series, the gold price series does not vary in a fixed level which indicates that the series is nonstationary in both mean and variance, exhibits upward and nonseasonal trends which reflect the ARIMA models. The hybridization of $\operatorname{ARIMA}(1,1,1)-\mathrm{GARCH}(0,2)$ revealed significant result at $1 \%$ significance level and satisfied the diagnostic checking including the heteroskedasticity test. The plotting of forecast and actual data exhibited the trend of forecast prices follows closely the actual data including for the simulation part of five days out-sample period. Consequently, the hybrid model of ARIMA $(1,1,1)$ $\operatorname{GARCH}(0,2)$ for the transformed data is given by

$$
\begin{aligned}
& y_{t}^{*}=0.274 y_{t-1}^{*}+0.726 y_{t-2}^{*}+\varepsilon_{t}-0.992 \varepsilon_{t-1} \quad, \varepsilon_{t} \sim \text { iid } N(0,1) \\
& \sigma_{t}^{2}=1.16 \times 10^{-5}+1.992 \sigma_{t-1}^{2}-1.025 \sigma_{t-2}^{2}
\end{aligned}
$$

Empirical results indicate that the proposed hybrid model ARIMA-GARCH has improved the estimating and forecasting accuracy by fivefold compared to the previously selected forecasting method. The findings suggest that combination of ARIMA (powerful and flexibility) and GARCH (strength of models in handling volatility and risk in the data series) have potential to overcome the linear and data limitation in the ARIMA models. Thus, this hybridization of ARIMA-GARCH is a novel and promising approach in gold price modeling and forecasting.

Keywords: $\quad$ ARIMA, GARCH, gold price forecasting, hybrid ARIMA-GARCH, Box-Cox transformation 


\section{INTRODUCTION}

Gold has been the most popular commodity as a healthy return investment due to its unique properties as a safe haven asset. Currency crises, inflation and instability economy in many countries since 1933 such as the Great Depression (1933), Asean crisis (1997-98) and global economic crisis (2008-2010) showed that gold being used as a hedge against inflation. According to Shafiee and Topal (2010), there is a significant negative relationship between inflation and the gold price over the last 40 years. Besides that, gold still an excellent store of value compared to other financial assets and commodities. By looking at the historical trend of gold price that are fluctuated based on global supply and demand and no risk-free, hence it is necessary to develop a model that reflects the pattern of the gold price movement since it become very significant to investors.

Gold prices are naturally time series data. Time series analysis develops a model for data series based on historical data and its past error. One of the widely used univariate time series models in many research practices is Box-Jenkins modelling. The Box-Jenkins modelling is one of the most powerful forecasting techniques available and it can be used to analyze almost any set of data (Christodoulos et al., 2010). In economics, Box-Jenkins modeling is well used in exchange rate forecasting, a basis model in gold price forecasting, and as a benchmark forecast model for gold and silver returns.

In many practical applications, the autoregressive integrated moving average (ARIMA) model is the most widely used Box-Jenkins models since it can handle nonstationary data. According to Shafiee and Topal (2010), the gold price followed a random walk and nonstationary characteristics. Therefore, ARIMA has been good potential to be a forecasting model for gold prices. ARIMA is one of the most important time series models used in financial market forecasting over the past three decades due to its statistical properties, accurate forecasting over a short period of time and ease of implementation (Khashei et al., 2009).

Despite the fact that the ARIMA is powerful and flexible, it is not able to handle the volatility and nonlinearity that are present in the data series. Gold as a well-established physical commodity has been actively traded spot on international markets since 1967. Since then, the gold market has been volatile. Previous studies showed that generalized autoregressive conditional heteroskedatic (GARCH) models are used in time series forecasting to handle volatility in the commodity data series including in the gold price volatility.

Combining models or hybrid the models can be an effective way to overcome the limitations of each components model as well as able to improve forecasting accuracy. In recent years, more hybrid forecasting models have been proposed applying Box-Jenkins models including an ARIMA model with GARCH to time series data in various fields for their good performance. Wang et al. (2005) proposed an ARMA-GARCH error model to capture the ARCH effect present in daily stream flow series. Zou et al. (2006) applied the ARIMA-GARCH model in forecasting internet traffic, while Chen et al. (2011) suggested ARIMA-GARCH model for short-time traffic flow prediction. In energy price forecasting, Tan et al. (2010) proposed a forecasting method that a combination of wavelet transform with ARIMA and GARCH models in predicting day-ahead electricity price. Meanwhile, Pham and Yang (2010) proposed the ARMA-GARCH procedures for estimating the machine health condition. In 2013, Liu and Shi applying ARMA-GARCH approaches to forecasting short-term electricity prices. Meanwhile, Liu et al. (2013) applied ARMA-GARCH-in-mean for wind speed forecasting. In the study, they explained the ARIMA-GARCH methodology as it is closed related the proposed model.

There are many forecast models used in forecasting gold price. The models which has been used recently are Box-Jenkins models (Miswan et al., 2013; Khashei et al., 2008), back propagation neural network (Yuan, 2012; Zhou et al., 2012; Parisi et al., 2008), system dynamics model (Tharmmaphornphilas et. al, 2012), varying-coefficient regression model (Zhang et al., 2011), data mining methods (Mustaffa \&Yusof, 2011), jump-and-dip diffusion (Shafiee and Topal, 2010), artificial intelligence models, multiple linear regression models (Ismail et al., 2009) or can be the hybrid of the above mentioned models (Khashei et al., (2009, 2008); Hadavandi et al., 2010; Asadi et al., 2012).

Although these models achieve a certain effect in forecasting gold price, there is no study that focuses on the performance of hybrid of ARIMA models with GARCH. In practice, the logarithmic function is used in transforming data in any financial data series. However in this study, the formula of Box-Cox transformation will be applied in the data transformation step. In line with these needs, this research is considered the pioneer in proposing a hybridization of the univariate time series modeling with volatility models incorporates with Box-Cox transformation in analyzing gold price. In this study, GARCH as the superior volatility model is applied to construct a hybrid model to overcome the linear limitations of ARIMA models by considering volatility in the forecast model in an attempt to yield more accurate forecast results. 


\section{TIME SERIES FORECASTING MODEL AND METHODOLOGY OF HYBRIDIZATION}

\subsection{ARIMA Models}

The autoregressive integrated moving average model of order $p$ and $q$, $\operatorname{ARIMA}(p, d, q)$ is suggest for the nonstationary and nonseasonality data series. Let $y_{t}$ and $\varepsilon_{t}$ be the observed value and random error at time period $t$, respectively; with $\mu$ is the mean of the model, $\varphi_{1}, \varphi_{2}, \ldots, \varphi_{p}$ are the autoregressive parameters with order $p, \theta_{1}, \theta_{2}, \ldots, \theta_{q}$ are the moving average parameters with order $q$, and $d$ is the order of differencing. The general form for $\operatorname{ARIMA}(p, d, q)$ that generates the time series with the mean $\mu$ has the form as in Eq. (1),

$$
\varphi_{p}(B)(1-B)^{d}\left(y_{t}-\mu\right)=\theta_{q}(B) \varepsilon_{t}
$$

where $\varphi_{p}(B)=1-\sum_{i=1}^{p} \varphi_{i} B^{i}, \theta_{q}(B)=1-\sum_{j=1}^{q} \theta_{j} B^{j}$ are polynomials in terms of $B$ of degree $p$ and $q, \nabla=(1-B)$, and $B$ is the backward shift operator.

ARIMA is one type of models in the Box-Jenkins modeling. The Box - Jenkins methodology includes four iterative steps of model identification, parameter estimation, diagnostic checking and forecasting. In identification step, data transformation is required to make the series stationary. The stationary process is a necessary condition in building an ARIMA model. When the observed time series presents trends and nonseasonal behavior, data transformation and differencing are applied to the data series in order to stabilize variance and to remove the trend before an ARIMA model is applied. In the Box-Jenkins modeling, the autocorrelation function (ACF) and the partial autocorrelation function (PACF) of the sample data are used in identifying the order of the time series model. The chosen model then is statistically checked whether it accurately describes the series. The model fits well if the $P$-value of its parameter is statistically significant, as well as its residuals are generally small, randomly distributed, and contain no useful information, where at this point, the model can be used for forecasting.

\subsection{GARCH Models}

For a univariate series, let

$$
y_{t}=\mu_{t}+a_{t}
$$

be a mean equation at time $t$, where $\mu_{t}$ is conditional mean of $y_{t}$ and $a_{t}$ is the shock at time $t$ and $a_{t}=\sigma_{t} \varepsilon_{t}$ where $\varepsilon_{t} \sim$ iid $N(0,1)$. Then $a_{t}$ follows a $\operatorname{GARCH}(r, s)$ model if

$$
\sigma_{t}^{2}=\alpha_{0}+\sum_{i=1}^{r} \alpha_{i} a_{t-i}^{2}+\sum_{i=1}^{s} \beta_{i} \sigma_{t-i}^{2}
$$

where $\sigma_{t}^{2}$ is the conditional variance of $y_{t}, \alpha_{0}>0$ and $\sum_{i=1}^{\max (r, s)}\left(\alpha_{i}+\beta_{i}\right)<1$. Note that $\alpha_{i}$ and $\beta_{i}$ are the coefficient of the parameters ARCH and GARCH, respectively. According to Pham \& Yang (2010), ACF and PACF of the residuals help to specify the GARCH orders, $r$ and $s$, respectively.

\subsection{Hybrid ARIMA-GARCH}

There is two-phase procedure in the proposed hybrid model of ARIMA and GARCH. In the first phase, the best of the ARIMA models is used to model the linear data of time series and the residual of this linear model will contain only the nonlinear data. In the second phase, the GARCH is used to model the nonlinear patterns of the residuals. This hybrid model which combines an ARIMA model with GARCH error components is applied to analyze the univariate series and to predict the values of approximation series (see Liu et al., 2013; Chen at al., 2011; Tan et al., 2010; Zou et al., 2006; Bollerslev and Wooldridge, 1992). In this procedure, the error term $\varepsilon_{t}$ of the ARIMA model is said to follow a GARCH process of orders $r$ and $s$. The methodology of this hybrid procedure is shown in Figure 1. 


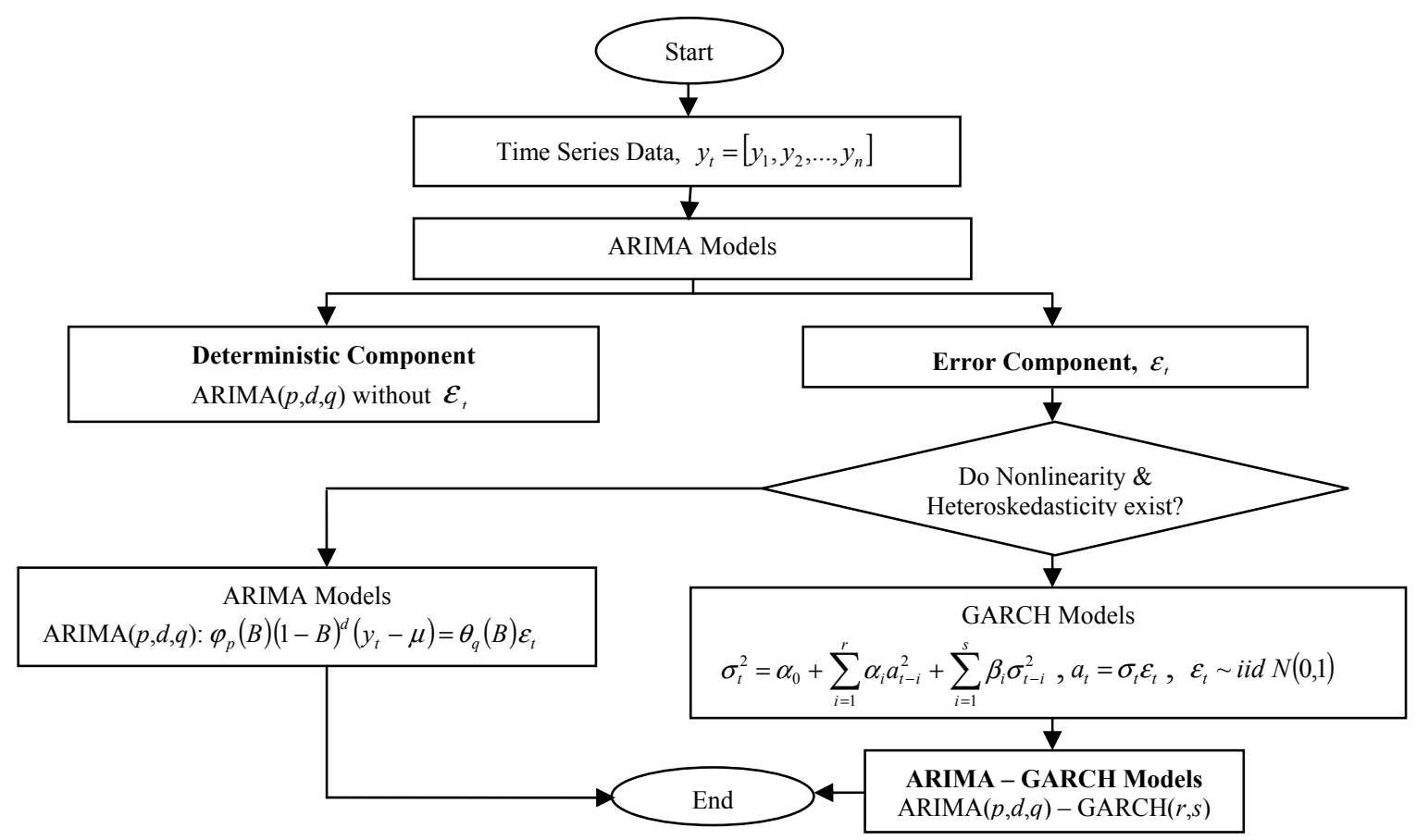

Figure 1. Flowchart of the procedure for hybridization of Box-Jenkins and GARCH models

\section{DATA TRANSFORMATION METHOD: BOX-COX TRANSFORMATION}

According to Osborne (2010), Box-Cox transformation represents a potential best practice whenever normalizing data or equalizing variance is desired, which it is envisioned as a panacea for simultaneously correcting normality, linearity and reducing homoskedasticity. The formula of the Box-Cox transformation is

$$
y_{t}^{*}= \begin{cases}\frac{y_{t}^{\lambda}-1}{\lambda}, & \text { for } \lambda \neq 0 \\ \log _{\mathrm{e}}\left(y_{t}\right), & \text { for } \lambda=0\end{cases}
$$

where $y_{t}$ is the actual data at the time $t, y_{t}^{*}$ is the transformed data at time $t$, and $\lambda$ is the minimum residual mean square error value. The transformation in Eq. (4) is valid only for positive series, $y_{t}>0$.

\section{MODELING OF GOLD PRICE}

\subsection{Data of Study}

In this study, a total of 40 daily gold price data series in USD/grams is used from $26^{\text {th }}$ November 2005 to $18^{\text {th }}$ January 2006 of 5-day-per-week frequencies. The data are divided into two parts: ( i) in-sample period for the first 35 observations; (ii) out-of-sample for the last five observations. The analysis is made on the same data series used by Asadi et al. (2012), Hadavandi et al. (2010), Khashei et al. (2009) and Khashei et al. (2008).

\subsection{Methodology and Analysis}

The first step of identification is to check the occurrence of a trend as well as seasonality in gold price movement by plotting in-sample series as shown in Figure 2. From the plotting, it can be seen that the gold price series does not vary in a fixed level which indicates that the series is nonstationary in both mean and variance, as well as exhibits an upward and nonseasonal trends.

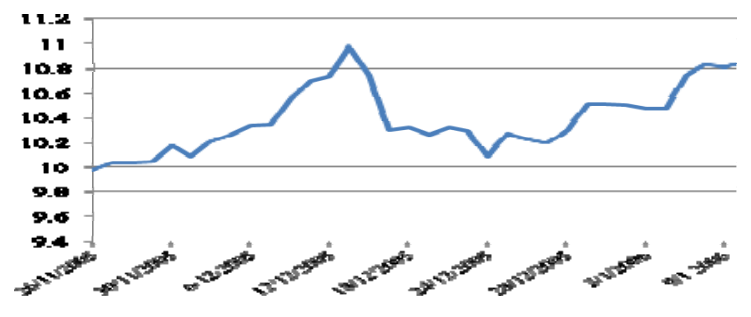

Figure 2. The in-sample gold price data series 
The observed series need to be transformed since it is nonstationary in variance. Using the rounded value of $\lambda=0.17$ given by Minitab, the Box-Cox transformation of $y_{t}{ }^{*}=\frac{y_{t}{ }^{0.17}-1}{0.17}$, where $y_{t}{ }^{*}$ is the transformed data and $y_{t}$ is the observed data, is applied to the gold price series. From the plotting of transformed data series, it can be seen that the series is less volatile, as the standard deviation of the transformed series decreased from 0.277 to 0.040 . However, the graph shows that the trend is still exist on the transformed series, which indicate that there is non stationarity in the mean. According to the unit root test, the $p$-value of 0.682 of Augmented Dickey-Fuller (ADF) test for the transformed series is strongly disagrees that the series is stationary. The correlograms of ACF and PACF of the transformed series that are slowly decaying also support the results. Thus, the transformed gold price series need to be differenced in order to remove the trend and obtain a stationary series.

The $p$-value $=0.000$ indicates the ADF $t$-statistic is significant, means the first differenced transformed series is stationary. The graph plotting of the first differenced transformed series illustrates the stationarity of the first order differenced transformed gold price series since most of the data are located around mean of zero. The stationarity of the first differenced series then supported by the correlogram patterns of ACF and PACF for the series, where the values are reduced drastically to zero. The Jarque-Bera test indicates that the differenced series is normally distributed at 5\% significance level. Thus, the first order difference for transformed gold prices series is used to find appropriate hybrid models of ARIMA-GARCH approaches. In this study, the maximum likelihood estimation (MLE) method is used in the parameter estimation. In the estimation stage, the values of Akaike information criterion (AIC) and Schwarz information criterion (SIC) are considered. In this context, the model with smaller AIC and SIC values are concluded to be the better estimation model.

Based on the correlograms of ACF and PACF for the first differenced transformed series and the residuals series, there are 40 possible model combinations between ARIMA and GARCH for $p=0,1,2, d=1$, $q=0,1,2, r=0,1$ and $s=0,1,2$. From the analysis conducted in the estimation stage, two of the ARIMAGARCH models show significant results. The results of AIC and SIC for those 2 models are given in Table 1 , show that the hybrid model of $\operatorname{ARIMA}(1,1,1)-\operatorname{GARCH}(0,2)$ produced the most negative values for both AIC and SIC, and significant at all levels of significance. Therefore, only the $\operatorname{ARIMA}(1,1,1)-\operatorname{GARCH}(0,2)$ is considered for the next diagnostic checking or cleaning data stage.

Table 1. The results of estimation stage of the possible ARIMA models.

\begin{tabular}{cccccc}
\hline Models & $\alpha=0.01$ & $\alpha=0.05$ & $\alpha=0.1$ & AIC & SIC \\
\hline ARIMA(1,1,1)-GARCH(0,2) & sig. & sig. & sig. & -5.194 & -4.922 \\
ARIMA(2,1,2)-GARCH(1,0) & not & not & sig. & -5.064 & -4.743 \\
\hline
\end{tabular}

The diagnostic checking is conducted to check the adequacy of the model considered where the test of LjungBox $Q$-statistic, heteroscedasticity test and the normality analysis are considered on the residuals of the model. The ACF and PACF of the squared standardized residuals for all the considered hybrid models show approximately zero, which is consistent with the insignificant Ljung-Box $Q$-statistic $p$-value that determine the models are adequate. The assumption of normality of the standardized residuals for all models is satisfied by the test of Jarque-Bera. Meanwhile, the ARCH-LM test on the residuals of the model indicates that the conditional heteroscedasticity is no longer present in the data series.

In the forecasting stage, the series of out-of-sample transformed data consists of five observations are used to obtain the forecast results. The prediction error is compared across models on the basis of two evaluation criteria commonly used in the previous literatures. The evaluation criteria are the mean absolute error (MAE) and mean square error (MSE). The best forecasting model is the one that generates the lowest prediction error of MSE and MAE. Let $n$ be the number of forecasts, $y_{t}$ and $\hat{y}_{t}$ are the actual and the predicted values of $y_{t}$ at time $t$, respectively. The evaluation criteria are given in Eq. (5) and Eq. (6).

$$
\begin{aligned}
\text { MAE } & =\frac{1}{n} \sum_{t=1}^{n}\left|y_{t}-\hat{y}_{t}\right| \\
\mathrm{MSE} & =\frac{\sum_{t=1}^{n}\left(y_{t}-\hat{y}_{t}\right)^{2}}{n}
\end{aligned}
$$


The prediction errors and forecast evaluations for the proposed model with all the considered models from previous studies are presented in Table 2. From the forecasting results, it can be concluded that ARIMA(1,1,1)-GARCH(0,2) outperform by five times better compare to the ten selected methods in the literatures in forecasting daily gold price. Consequently, the hybrid model of $\operatorname{ARIMA}(1,1,1)-\operatorname{GARCH}(0,2)$ for the transformed data is given by Eq. (7).

$$
\begin{aligned}
& y_{t}^{*}=0.274 y_{t-1}^{*}+0.726 y_{t-2}^{*}+\varepsilon_{t}-0.992 \varepsilon_{t-1} \quad, \varepsilon_{t} \sim \text { iid } N(0,1) \\
& \sigma_{t}^{2}=1.16 \times 10^{-5}+1.992 \sigma_{t-1}^{2}-1.025 \sigma_{t-2}^{2}
\end{aligned}
$$

Table 2. The performance of the proposed model compared to some selected forecasting models

\begin{tabular}{lcc}
\multicolumn{1}{c}{ Models } & MAE & MSE \\
\hline ARIMA [Khashei et al., 2008] & 0.105 & 0.017 \\
Chen's fuzzy time series (first order) [Khashei et al., 2008] & 0.297 & 0.116 \\
Chen's fuzzy time series (second order) [Khashei et al., 2008] & 0.292 & 0.111 \\
Yu's fuzzy time series [Khashei et al., 2008] & 0.297 & 0.116 \\
Artificial neural networks (ANNs) [Khashei et al., 2008] & 0.170 & 0.034 \\
ANFIS [Khashei et al., 2008] & 0.081 & 0.013 \\
Hybrid of ARIMA, ANN and fuzzy [Khashei et al.,2009] & 0.097 & 0.012 \\
PSO-based time series [Hadavandi et al., 2010] & 0.047 & n.a \\
OLS method [Hadavandi et al., 2010] & 0.098 & n.a \\
PSOARIMA [Asadi et al., 2012] & 0.0439 & 0.005 \\
ARIMA-GARCH (proposed model) & 0.008 & 0.0001 \\
\hline
\end{tabular}

*n.a not available

The following Figure 3 is the forecasting results of $\operatorname{ARIMA}(1,1,1)-\operatorname{GARCH}(0,2)$ with \pm 2 standard errors for daily gold prices from $26^{\text {th }}$ November 2005 to $18^{\text {th }}$ January 2006. Graphically, the figure shows the promising performance of the hybrid model in forecasting daily gold price series which is the trend of forecast prices follows closely the actual data including for the simulation part of five days out-sample period. The comparison values between actual data of daily gold price and forecast price using the proposed model for the five-days out-sample period is given by Table 3 .

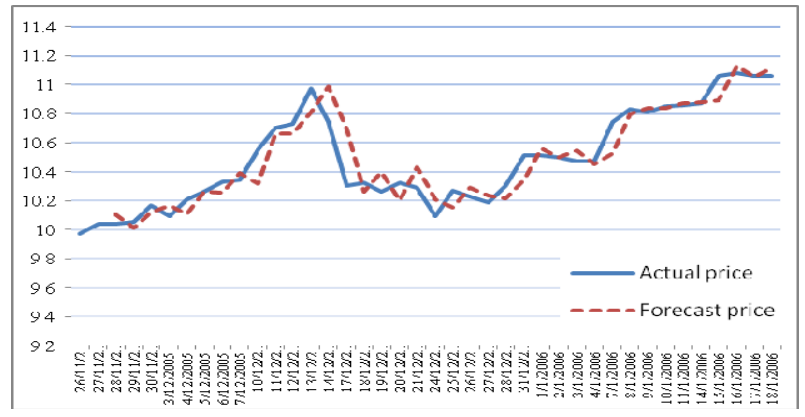

Figure 3. Graph of the actual data and forecast data using $\operatorname{ARIMA}(1,1,1)-\mathrm{GARCH}(0,2)$
Table 3. The comparison between actual price and forecast price for out-sample period

\begin{tabular}{ccc}
\hline Date & $\begin{array}{c}\text { Actual } \\
\text { price }\end{array}$ & $\begin{array}{c}\text { Forecast } \\
\text { price }\end{array}$ \\
\hline 14 January & 10.87 & 10.88 \\
15 January & 11.06 & 10.89 \\
16 January & 11.08 & 11.13 \\
17 January & 11.06 & 11.06 \\
18 January & 11.06 & 11.11 \\
\hline
\end{tabular}

\section{DISCUSSION AND CONCLUSION}

This study examined the performance of hybrid of the most powerful univariate time series, ARIMA models with the superior volatility models, GARCH in analyzing and forecasting daily gold price data series. The Box-Cox formula is used in the data transformation step to address non stationarity in variance. The empirical results of 40-day gold price data series indicate that the hybrid ARIMA $(1,1,1)-\operatorname{GARCH}(0,2)$ model provide the optimum results and effectively improved estimating and forecasting accuracy compared to the ten previous methods of forecasting in literatures. In conclusion, the complete combination of powerful and flexibility of ARIMA and the strength of GARCH models in handling volatility and risk in the data series as well as to overcome the linear and data limitation (see Khashei et al., 2009) in the ARIMA models made the combination of ARIMA-GARCH as a new potential approach in analyzing and forecasting daily gold price. 
Yaziz et al., The performance of hybrid ARIMA-GARCH modeling in forecasting gold price

\section{REFERENCES}

Asadi, S., Tavakoli, A. \& Hejazi, S. R. (2012). A new hybrid for improvement of auto-regressive integrated moving average models applying particle swarm optimization. Expert Systems with Applications, 39, $5332-5337$.

Bollerslev, T. \& Wooldridge, J. M. (1992). Quasi-maximum likelihood estimation and inference in dynamic models with time-varying covariances. Econometric Reviews, 11(2), 143-172.

Chen, C., Hu, J., Meng, Q. \& Zhang, Y. (2011). Short-time traffic flow prediction with ARIMA-GARCH model. 2011 IEEE Intelligent Vehicles Symposium (IV), Baden-Baden, Germany, June 5-9.

Christodoulos, C., Michalakelis, C. \& Varoutas, D. (2010). Forecasting with limited data: Combining ARIMA and diffusion models. Technological Forecasting \& Social Change, Article in Press.

Hadavandi, E., Ghanbari, A. \& Abbasian-Naghneh, S. (2010). Developing a Time Series Model Based On Particle Swarm Optimization for Gold Price Forecasting. $3^{\text {rd }}$ International Conference on Business Intelligence and Financial Engineering (BIFE 2010), IEEE computer society, 337-340.

Ismail, Z. Yahya, A. \& Shabri, A. 2009. Forecasting gold prices using multiple linear regression method. American Journal of Applied Sciences, 6(8), 1509-1514.

Khashei, M., Hejazi, S. R., Bijari, M. (2008). A new hybrid artificial neural networks and fuzzy regression model for time series forecasting. Fuzzy Sets and Systems, 159:769-786.

Khashei, M., Bijari, M., \& Raissi, G.H.A. (2009). Improvement of auto-regressive integrated moving average models using fuzzy logic and artificial neural networks (ANNs). Neurocomputing, 72, 956-967.

Liu, H. \& Shi, J. (2013). Applying ARMA-GARCH approaches to forecasting short-term electricity prices. Energy Economics, 37, 152-166.

Liu, H., Shi, J. \& Qu, X. (2013). Empirical investigation on using wind speed volatility to estimate the operation probability and power output of wind turbines. Energy Conversion and Management, 67, 8-17.

Miswan, N. H, Ping, P.Y. \& Ahmad, M. H. (2013). On parameter estimation for Malaysian gold prices modelling and forecasting. International Journal of Mathematical Analysis, 7 (21-24), 1059-1068.

Mustafa, Z. \& Yusof, Y. (2011). Optimizing LSSVM using ABC for non-volatile financial prediction. Australian Journal of Basic and Applied Sciences, 5(11), 549-556.

Parisi, A., Parisi, F., \& Diaz, D. (2008). Forecasting gold price changes: Rolling and recursive neural network models. Journal of Multination Financial Management, 18, 477-487.

Pham, H. T. \& Yang, B-S. (2010). Estimation and forecasting of machine health condition using ARMA/GARCH model. Mechanical Systems and Signal Processing, 24, 546-558.

Shafiee, S. \& Topal, E. (2010). An overview of global gold market and gold price forecasting. Resources Policy, 35, 178-189.

Tan, Z., Zhang, J., Wang, J. \& Xu, J. (2010). Day-ahead electricity price forecasting using wavelet transform combined with ARIMA and GARCH models. Applied Energy, 87, 3603-3610.

Tharmmaphornphilas, W., Lohasiriwat, H. \& Vannasetta, P. (2012). Gold price modeling using system dynamics. Engineering Journal, 16(5),57-67.

Wang, W., Van Gelder, P. H. A. J. M., Vrijing, J. K. \& Ma, J. (2005). Testing and modeling autoregressive conditional heteroskedasticity of streamflow processes. Nonlinear Processes in Geophysics, 12, 55-66.

Yazdani-Chamzini, A., Yakhchali, S. H., Volungeviciene, D. \& Zavadskas, E. K. (2012). Forecasting gold price changes by using adaptive network fuzzy inference system. Journal of Business, Economics and Management, 13(5), 994-1010.

Yuan, G. (2012). Study on gold price forecasting technique based on neural network optimized by GA with projection pursuit algorithm. Journal of Convergence Information Technology, 7 (18), 558-565.

Zhang, Q., Ma, J. \& Wang, Y. (2011). Study on forecasting of gold price based on varying-coefficient regression model. Key Engineering Materials, 467-469, 1398-1403.

Zhou, S., Lai, K. K. \& Yen, J. (2012). A dynamic meta-learning rate-based model for gold market forecasting. Expert System with Applications, 39, 6168-6173.

Zou, B., He, D. \& Sun, Z. (2006). Traffic predictability based on ARIMA/GARCH model. Modeling and Simulation Tools for Emerging Telecommunication Networks, 101-121. 